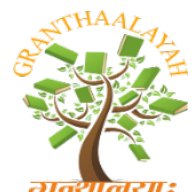

INTERNATIONAL JOURNAL OF RESEARCH GRANTHAALAYAH A knowledge Repository

Science

\title{
OBSTETRIC RISKS IN PREGNANT WOMEN ATTENDED AT THE GYNECOLOGICAL OBSTETRIC UNIVERSITY HOSPITAL OF GUAYAQUIL
}

\author{
Obsta Vicky Narea Morales MSc ${ }^{1}$, Dr. Jorge Daher Nader PhD ${ }^{1}$, Obsta. Katherine \\ Rodríguez $^{1}$, Obsta Gabriel Jiménez ${ }^{1}$, Obsta. Cynthia Carolina Armijo Baño ${ }^{1}$ \\ ${ }^{1}$ Trachers of the Careers of Obstetric of The University of Guayaquil
}

\begin{abstract}
The word risks is derived from the Greek word riscare, that is to say, they dare, the concept of risks is associated with several possibilities and a predisposing risk factor in pregnancy which seems to identify the degrees of vulnerability during the periods of pregnancy, childbirth, puerperium, the present research work aimed to determine the main obstetric risks in pregnant women of the Hospital Gineco Obstétrico Universitario de Guayaquil, Guayas - Ecuador from January - June 2019. The study design is non-experimental, prospective, in the methodology from the investigation, the data collection form was used, an ad hoc form was prepared. The sample was made up of 407 nulliparous and multiparous pregnant women, who met the inclusion and exclusion criteria. The following results were obtained: preeclampsia with $36 \%$, diabetes $29 \%$, gestational hypertension $20 \%$, preterm labor $6 \%$, premature rupture of membranes $3 \%$ and the placenta praevia $1 \%$. In conclusion, the obstetric risks observed in the present study, the highest percentage is preeclampsia with $36 \%$ and the lowest percentage placenta previa with $1 \%$.
\end{abstract}

Keywords: Obstetric Risk: Perinatal; Pregnant; 35 years.

Cite This Article: Obsta Vicky Narea Morales MSc, Dr. Jorge Daher Nader PhD, Obsta. Katherine Rodríguez, Obsta Gabriel Jiménez, and Obsta. Cinthya Baño. (2020). “OBSTETRIC RISKS IN PREGNANT WOMEN ATTENDED AT THE GYNECOLOGICAL OBSTETRIC UNIVERSITY HOSPITAL OF GUAYAQUIL." International Journal of Research Granthaalayah, 8(3), 238-244. https://doi.org/10.5281/zenodo.3734300.

\section{Introduction}

The word risks is etymologically derived from the word riscare, that is, they dare. The concept of risks is associated with various possibilities and the link between a risk factor and harm is not always explained. Specifically, the concept of risk in pregnancy seems to identify the degrees of vulnerability during the periods of pregnancy, childbirth, postpartum and the life of the child in its first year. The obstetric risk classification is divided into 3 groups; • Low risk or Type I.- Those pregnant women who are in good health in the current pregnancy and have no history of 
complications in previous pregnancies or perinatal death, but there are social biopsy risks that predispose them to subsequent complications such as: Clinically and / or radiologically identified pelvic abnormality, maternal height: $<1.45$ meters. Heart disease 1: that does not limit your physical activity, Unfavorable socioeconomic conditions. Insufficient control of pregnancy: $<4$ prenatal visits or 1 visit after week 20 of gestation, extreme age $<16$ or $>35$ years, unwanted pregnancy, previous sterility: two years or more, habitual smoker, date of uncertain last menstruation, pregnant with Rh negative, great multiparity: 5 or more fetuses of at least 28 weeks, first trimester hemorrhage, Rh incompatibility, obesity, intergenetic period less than 12 months or greater than 5 years, mental and / or physical disability, vulnerable groups. • High risk or Type II.Those pregnant women who are in good health in the current pregnancy, without a history of perinatal death, but have a gyneco-obstetric history such as: Severe anemia $(\mathrm{Hb}<9 \mathrm{~g} / \mathrm{dl}$ or hematocrit $<25 \%$ ) Heart disease 2: patient forced to slightly limit their physical activity, previous uterine surgery, gestational diabetes, twin pregnancy, prolonged pregnancy: more than 42 weeks of gestation, endocrinopathy (thyroid, adrenal, hypothalamic), 2nd or 3rd hemorrhage trimester, hydramnios or oligoamnios.

Unfavorable obstetric history: two or more abortions, one or more premature, dystocic deliveries, mental or sensory deficiency of probable obstetric origin, history of growth retardation, maternal infection: TORCHES, morbid obesity, mild pre-eclampsia: diastolic BP greater than or equal to $90 \mathrm{mmhg}$, suspicion of fetal malformation, abnormal presentation: confirmed after week 38 of gestation, malignancies, tumors, chronic diseases. - Very high irrigation or type III.- Those pregnant women who are in good health in the current pregnancy with a history of perinatal death; or pregnant women who have complications in the current pregnancy with or without a history of perinatal death; as well as pregnant women who have underlying illnesses, such as: threatened premature birth: before week 37, heart disease 3 or 4: patient forced to a marked limitation of their physical activity or in which their decompensation, drug addiction or alcoholism, multiple gestation (3 or more), cervical incompetence, confirmed fetal malformation, recurrent perinatal death, placenta previa, RCI, RPM, and HIV. TEAR AND INJURIES IN THE SOFT DELIVERY CHANNEL. TEAR OF THE UTERINE NECK. - Virtually all deliveries cause small tears to the cervix. This explains why the external service does not recover the circular shape that it has in the nullipara after childbirth, the tears can be only bilateral or radiated. The most frequent are observed in the lateral angles towards 3 or 9 o'clock, generally only affecting the intravaginal portion of the neck, but sometimes it can extend to the supra-vaginal area and even affect the uterine body. Producing a uterine. Cervical tears extend toward the parameters or even affect the bladder. The origin is attributed to mechanical problems during childbirth.

Tear of The Vagina. - Tears of the vagina caused by childbirth rarely occur in isolation, in general, usually complicate the perine. They can be varied, with longitudinal or transverse direction, sometimes they appear on several faces of the vagina, with an infractive shape. The etiological factors are, vaginal hyperdistension by a large head, an excessively rapid decrease and clinically manifested by the presence of hemorrhage. It begins after the fetus is expelled, but sometimes this bleeding is not verified until after the placental expulsion. Then it will be seen to persist, even though the uterus is well contracted. The definitive diagnosis is made by direct visualization of the vagina with adequate leaflets. 
Tear Of The Perine.- They are the most frequent of all the injuries that can occur in the soft canal of childbirth. Those that affect the mucosa of the rectum are classified as grade 1 and grade 2 and grade 3 .

Vulvovaginal Hematoma. - Occasionally as a result of the trauma of childbirth, a rupture of vessels occurs, it can produce a hematoma that extends under the skin of the vulva, vagina. This is possible without a skin or vagin defect, it can also occur after a defective suture of a perineal tear or an episiotomy.

\section{Perinatal Risks}

It is the threat of injury or death to which they are exposed from 28 weeks of gestation to the 7 th day of birth, some products of conception in which certain pre-disposing factors appear. The perinatal period is one of the great challenges facing health services today, because pregnancy is one of the stages of greatest morbidity and mortality worldwide, it can be controlled through detection and management timely risk in pregnant patients, for this the evolution of the pregnancy will be evaluated, the mother is prepared for the delivery and the upbringing of her child, for this reason the prenatal control must be effective and efficient, ensuring that the pregnancy ends with a birth of a healthy child and without deterioration of the mother's health in the face of any pathophysiological process. With the medical checkup, you will avoid risks for the mother and the fetus. Newborn respiratory distress syndrome (RDS) It is a picture that begins shortly after birth or in the first hours of life and is characterized by tachypnea, cyanosis, moaning, subcostal retraction, and variable degrees of oxygenation compromise. It is already corrected - Transient tachypnea of the newborn (NRT)

It is a neonatal respiratory disease with an acute evolution, self-limited and benign, caused by the retention, after birth, of fetal lung fluid, which under normal conditions is removed thanks to the fact that the alveolar epithelium changes from secreting chlorine to sodium absorption and Prostaglandins dilate the lymphatic vessels and promote the passage of fluid into the pulmonary circulation, which is fully established after the first breath. Prematurity It is defined by the World Health Organization (WHO) as the birth that occurs before the completion of 37 weeks or before 259 days of gestation. It is subdivided into extremely premature $<28$ weeks $(5.2 \%$ of all $<37$ weeks, very premature $28-31$ weeks $(10.4 \%$ of all $<37$ weeks; and moderately premature $32-36$ weeks $(84.3 \%$ of all $<37$ weeks Among these moderately premature infants, a subgroup called "late preterm infants" (34-36 weeks) has been classified. - Risk of compromised fetal well-being Suspected or confirmed disturbance of metabolic status caused by a rapidly evolving variation in maternal-fetal exchanges, disrupting fetal homeostasis and causing hypoxia, hypercapnia, hypoglycemia, and acidosis, leading to sequelae injury and death. It is clinically manifested by alterations in fetal heart rate and expulsion of meconium, and the evolution of the clinical picture depends on sweating, the severity and the capacity of the fetal compensatory mechanisms. Intrauterine growth restriction Fetal growth below the 10th percentile on a selected intrauterine growth curve. Perinatal asphyxia. - It is defined as injuries to the fetus or the newborn, caused by hypoxia and ischemia of several organs of sufficient magnitude to produce biochemical and / or functional changes. It occurs when the placenta separates from the uterus too soon (placental abruption), there are other problems with the placenta that can also cause fetal death, infections, disorders that they reduce the flow of blood (and consequently oxygen and nutrients) to the fetus and bleeding. An obstetric complication is rare but potentially devastating, not only for the mother- 
child pair, but also for your family and the healthcare professionals responsible for your care. In its most extreme form, obstetric complications can lead to the death of the woman or child during pregnancy or the puerperium. (MSP, 2016) The age of the woman as a risk factor for maternal, neonatal, and fetal mortality indicates a higher morbidity in maternal and neonatal mortality, with a higher incidence in women aged 35 or over. (Villalva, 2019) Complications in pregnancy, childbirth or the puerperium can occur due to multiple conditions, which are later classified into direct, indirect or incidental maternal deaths. Pregnancy in pregnant women over 35 years of age is classified as a high-risk pregnancy. This research work is justified in order to determine the obstetric and perinatal risks in pregnant women over 35 years old, it was carried out at the Gynecological Obstetric Pediatric University Hospital of Guayaquil in the emergency area and outpatient consultation, it is considered that the investigation help prevent, timely detect obstetric risks; to decrease the fetal maternal morbidity and mortality rate. The research is pertinent because it will benefit pregnant women in marginal urban sectors, by preventing obstetric risks; by giving them objective information based on protocols from the Ministry of Public Health; This will contribute to improving this health problem; This study was carried out from January to June 2019. Why should this research work be done? To avoid obstetric and perinatal risks in women over 35 years of age, who are considered a high-risk pregnancy, the work has a methodological utility, which is why I decided to carry out this research work and will serve for future research.

The objective is to determine obstetric risks in pregnant women over 35 years of age, attended at the Gyneco Obstetric University Hospital of Guayaquil in the period January - June 2019.

\section{Materials and Methods}

This research work corresponds to a non-experimental cross-sectional study, it is inductive Analytic.

The methodology is of non-experimental, prospective, descriptive, quantitative design, the population is 1,230 and the sample consisted of 407 pregnant women who met the inclusion and exclusion criteria.

The inclusion criteria included pregnant women with obstetric risk, women over 35 years of age, at the Hospital Universitario Pediátrico de Guayaquil in the study period.

In the exclusion criteria, pregnant women whose delivery is caesarean are excluded, patients who are not at obstetric risk and do not meet the inclusion criteria are excluded.

\section{Results}

1.- Obstetric Risk in hospital Universitario de Guayaquil.

Table 1: Obstetric Risk

\begin{tabular}{|l|c|c|}
\hline \multicolumn{3}{|c|}{ Table No 1 } \\
\hline \multicolumn{1}{|c|}{ Obstetric Risk } & Frequencies & PORCENTAJE \\
\hline Gestational hypertension. & 82 & $20 \%$ \\
\hline Hellp síndrome. & 6 & $2 \%$ \\
\hline
\end{tabular}




\begin{tabular}{|l|c|c|}
\hline Diabetes & 119 & $29 \%$ \\
\hline Preeclampsia & 146 & $36 \%$ \\
\hline RPM & 13 & $3 \%$ \\
\hline Double pregnancy & 11 & $3 \%$ \\
\hline Previous placenta. & 2 & $1 \%$ \\
\hline Preterm labor. & 26 & $6 \%$ \\
\hline TOTAL & 407 & $100 \%$ \\
\hline
\end{tabular}

Analysis and interpretation.- In table \# 1 Obstetric Risk, It is evident of the total of 407 pregnant women object of our study, preeclampsia with a frequency of 146 patients representing $36 \%$, diabetes $29 \%$, gestational hypertension $20 \%$, preterm labor $6 \%$, premature rupture of membranes $3 \%$ and the placenta with $1 \%$ patients representing the lowest percentage with $1 \%$.

\section{Results}

Table 2: Distribution of obstetric risk cases according to age.

\begin{tabular}{|l|c|c|}
\hline Age Pregnancy & Frequencies & Porcentaje \\
\hline 35- 40 años & 328 & $81 \%$ \\
\hline $41-45$ años & 74 & $18 \%$ \\
\hline $46-50$ años & 2 & $0,35 \%$ \\
\hline $51-55$ años & 2 & $0,35 \%$ \\
\hline 59 years onwards & 1 & $0,30 \%$ \\
\hline TOTAL & 407 & $100 \%$ \\
\hline
\end{tabular}

Analysis and interpretation.- In the table \# 2 we observe It observes that of the total of 407 pregnant women we can observe that 200 pregnant women object of our study correspond to women older than 35 years of age, 328 pregnant women with ages between 35 - 40 years with a percentage of $81 \%$.

3.- $i$ In which trimesters of Pregnancy are the Obstetric risks presented in pregnant women over 35 years of age?

Table 3: Trimesters of pregnancy.

\begin{tabular}{|l|c|c|}
\hline Trimesters of pregnancy. & Frequencies & Percentaje \\
\hline 1 Trimesters & 0 & $0 \%$ \\
\hline 2 Trimesters & 190 & $47 \%$ \\
\hline 3 Trimesters & 217 & $53 \%$ \\
\hline TOTAL & 407 & $100 \%$ \\
\hline
\end{tabular}

Analysis and interpretation.- In table \# 3 the table of the trimesters of pregnancy of pregnant women with obstetric risks over 35 years of age is observed, it is observed in the third trimester with a frequency of 217 , which represents the highest percentage with $53 \%$, followed by 190 of frequency that represents $47 \%$. 


\section{Conclusion}

At the end of the following investigative work, the following could be concluded:

1) It is concluded that the highest percentage of maternal obstetric risks is represented by preeclampsia, followed by diabetes.

2) Of the total sample 407, pregnant women object of our study, the maternal obstetric risks correspond to preeclampsia, gestational hypertension, eclampsia, diabetes.

3) From the total of the sample object of this study, the ages between 35 - 40 years correspond to the majority group.

4) It is concluded that, from the graphic representation of the trimesters of pregnancy of pregnant women with obstetric risks over 35 years of age, the third trimester represents the highest percentage.

Recommendations The following is recommended: 1.- The main recommendations are aimed at preventing maternal obstetric risks. 2.- Promote the creation of an educational intervention program for pregnant women aged 35 and over. 3.- Encourage pregnant women to go to prenatal checkups to identify obstetric risk factors. 4.- Creation of a pregnant club in health centers, where all pregnant women are summoned once a month to explain sexual and reproductive health issues. 5.- Pregnant women are recommended to have healthy nutrition during pregnancy. 6.- It is proposed to implement an educational intervention program on obstetric and perinatal risks to prevent morbidity and mortality in the Gyneco Obstetric Pediatric University Hospital of Guayaquil.

\section{References}

[1] Arboleda, R. (2016). Pregnant obstetric risk factors attending Delfina Torres de Esmeraldas hospital. 63.

[2] cristinage. (2014). Obstetric and perinatal complications in pregnant women over 35 years of age. 80 .

[3] Gutierrez. (2014). Obstetric and perinatal complications in pregnant women over 35 years of age. 60.

[4] Morales. (2016). Perinatal Obstetric Risks in pregnant women over 35 years of age, treated at the San Vicente de Paul hospital 2016. 79.

[5] MSP. (2016). Score Guide breast keys and obstetric risks. MSP protocol. 94.

[6] MSP. (2016). Score breast obstetric keys 2016 MSP Protocol. 94.

[7] Nolasco. (2015). Perinatal finding of pregnancies in advanced maternal age. 40.

[8] Villalva, B. (2019). Obstetric risks in pregnant women with extreme ages Hospital Universitario Guayaquil. 64.

[9] Comprehensive care protocol for adolescents 2009 MSP.

[10] National Statistics Institute (INEC). Youth Fertility in Chile 2007.

[11] Molina M, Peña I, Quiroz M, Pregnancy, Social Work Magazine, 2005.

[12] WILLIAMS. (s.f.). Gynecology - Williams Obstetrics. 24 edition, 1500.

[13] University of Chile. Proposal of public policies for the prevention of pregnancy in adolescence. Public Policy Analysis Center, Universidad de Chile 2006.

[14] Lankoande J. Quedraogo CM, tieba B. Maternal mortality in adolescents at the university hospital of Quagadougu. Rev Med Brux 2005. 
[15] Latin American Federation of Obstetric and Gynecological Societies (Flasog) committee on sexual and reproductive rights.

[16] Valdes Dacal S, Essien J, Bardales Mitac J, Saavedra Moredo D, Bardales Mitac Pregnancy in adolescence, Incidence, risks and complications. RCOG.

[17] Jose Antonio Usandizaga Beguristain and Pedro de la Fuente Perez of Obstetrics and Gynecology, anomalous delivery October 12, 2011 4th edition.

[18] F. Gary Cunningham, Kenneth j. Leveno, Stven L. Bloom, John C. Hauth, Dwight J. Rouse, Catherine Spong Williams Obstetrics 23rd Edition.

[19] Reyes Arboleda Paulett Stefania Obstetric risk factors in pregnant women who go to the Delfina Torres de Esmeraldas hospital. Pontificia Universidad Católica del Ecuador headquarters Esmeraldas school of nursing Esmeraldas, 2016. Pg. 63.

${ }^{*}$ Corresponding author.

E-mail address: Vicky.naream@ug.edu.ec/Jorge.dahern@ug.edu.ec/Katherine.rodriguezb@ug.edu.ed Gabriel Jimenezv@ug.edu.ec 